\title{
Provision of Voltage Ancillary Services through Enhanced TSO-DSO Interaction and Aggregated Distributed Energy Resources
}

\author{
Anastasios Oulis Rousis, Member, IEEE, Dimitrios Tzelepis, Member, IEEE, Yousef Pipelzadeh, Member, IEEE, \\ Goran Strbac, Member, IEEE, Campbell D. Booth, and Timothy C. Green, Fellow, IEEE
}

\begin{abstract}
The electrical energy generated from renewable energy resources connected to transmission and distribution systems and the displacement of synchronous generators continues to grow. This presages a paradigm-shift away from the traditional provision of ancillary services, essential to ensure a robust system, from transmission-connected synchronous generators towards provision from synchronous and non-synchronous generation (including distribution-connected resources). Given that the available resources at the disposal of system operators are continuously increasing, the flexibility for operating the network can be enlarged. In this context, this paper introduces a dedicated voltage ancillary services strategy for provision of reactive power. A main feature of the proposed strategy is that it is technology-neutral, unlike existing ones that are focused on synchronous generators. The system need for voltage stability is placed at the core of this strategy, which is translated into a requirement for reactive power provision. The proposed strategy achieves, through the combined utilization of distributed generation and traditional resources, to defer the investments in reactive compensating equipment. Dynamic and transient studies are conducted to demonstrate the technical benefits of the strategy, while its practical feasibility is also validated through hardware-in-the-loop testing.
\end{abstract}

Index Terms-Ancillary services, Voltage stability, Reactive power provision, TSO-DSO interaction, Hardware-in-the-loop.

\section{INTRODUCTION}

D ECENTRALIZATION and digitization are rapidly transforming the energy sector. Increasingly popular, distributed generation (DG), including photovoltaic (PV) plants, wind farms (WFs) and energy storage systems (ESS), is disrupting the traditional top-down philosophy of power systems [1]. This fundamental shift has implications on how the network is being operated. In addition to being predictable and controllable, large, synchronous power stations have historically provided ancillary services (AS) to the transmission system operators (TSOs) for a reliable and secure power system [2]. In contrast, it is much harder to predict the output of renewable technologies and additionally TSOs have limited visibility or control over these generators when connected to the distribution network [3]. As such, AS from DGs have very rarely been procured by TSOs. In response to this transformation, TSOs have lately invested significant amounts of capital in compensating devices (e.g. reactors and capacitors) to account for the AS that synchronous generators (SGs) would

This article outlines the results of a collaborative work conducted between Imperial College London, UK, University of Strathclyde, Glasgow, UK and Manitoba Hydro International, MB, Canada. This research was supported partly by IDLES Project UK (EPSRC Grant EP/R045518/1), Power Potential Project UK and by the PHOENIX Project UK (SPTEN03). otherwise provide, and eventually maintain the transmission system within acceptable stability and compliance margins. These measures are, however, not considered to be costeffective solutions, hence TSOs are currently looking into exploiting the untapped resources available in the distribution system [4].

The various ancillary services, usually deployed by TSOs, include voltage stability, frequency response, black-start capability, and so on. In the interest of voltage security, TSOs currently utilize either synchronous generators, when present, or capacitors, static VAr compensators (SVCs) and static compensators (STATCOMs) to regulate the reactive power flows in the transmission system. Even though these devices exhibit effective performance in maintaining voltage stability, they are characterized by significant capital costs suggesting that sole use of these resources can be economically ineffective [5].

To this end, this paper argues that the portfolio of resources (i.e. a combination of transmission and distribution-connected resources) providing services should increase and solutions should initially be sought in existing assets that can prove to be equally effective and more cost-saving. The paper, thus, identifies and classifies the equipment with a capability to support the system when voltage instabilities arise. To achieve this, a strategy for provision of voltage ancillary services (VAS) is designed, implemented and validated. The technical benefits of the proposed VAS are demonstrated with the help of appropriate dynamic phasor-domain and electromagnetic transient simulations, and the technical feasibility for implementation in TSO-DSO systems is validated using real-time simulation and hardware-in-the-loop (HIL) testing.

The proposed strategy aims at being technology-neutral; i.e. it attempts to interpret the needs of the system for reactive power into suitable command actions involving all equipment currently existing in transmission and distribution systems, as per their capability. This is supported by the fact that in coming years it is anticipated that no single technological solution mitigating all system challenges will exist, and as such the future power grid should amass the capability of all available solutions. This will inevitably lead to an increased utilization of resources traditionally not being exploited. Especially, the utilization of reactive power from distributionconnected resources (DCR), which have the inherent capability to enable bi-directional reactive power flow [6], will be brought forward. It is worth noting that the technical background in this area, according to the literature review, is still in immature stages. Most of the AS frameworks currently 
adopted are not technology-neutral, and there have only been very recently a few academic efforts to enhance such strategies with modern technologies [7], [8], [9]. However, even in these cases, the proposed strategies are generic without directly dealing with voltage considerations.

Specifically, [7] proposes TSO-DSO frameworks for provision of AS. Appropriate market models are developed to enhance TSO-DSO coordination for the provision of AS, but the technical feasibility of such schemes is not assessed through simulations since the focus is on the commercial arrangements. Additionally, [7] includes very little information on voltage control (i.e. the focus is on frequency-related AS), which means that no VAS are described. The work reported in [8] sets the basis for design of various types of AS as dictated by the changes in the power sector. The objective of [8] is to propose a way for designing AS frameworks, hence defines three basic elements. These elements are taken into account in Section II-D for designing the proposed VAS. The authors of this paper are also focused on the commercial arrangements for AS provision (i.e. design variables including products description, activation times, acquisition methods, etc.). Two example frameworks are given to demonstrate how modern AS frameworks should be designed, but once again they are targeting frequency control issues (i.e. frequency containment reserves and reserves for other purposes). Additionally, there is no technical study demonstrating the technical feasibility. Further work described in [9] discusses the shortfalls of the current AS framework of Electric Reliability Council of Texas, and describes the features of a technologyneutral framework to be deployed in the future. However, the focus is also on frequency control issues.

\section{A. Limitations of Existing Schemes and Contributions of the Proposed Framework}

The performed literature review has indicated that most of the existing papers focus on control schemes based on a limited number of technologies, typically transmission-connected assets, while the distributed assets found in the distribution network are disregarded towards provision of voltage ancillary services. On the contrary, the proposed solution combines most of the available technologies on the distribution level and takes into account the capability of each resource to provide voltage support. To the authors best knowledge, such work has not been presented elsewhere. A comparative review of recent solutions proposed in the literature is presented in Table I.

This paper introduces a dedicated, technology-neutral strategy for provision of voltage ancillary services. The proposed framework considers a holistic suite of assets with reactive power capability and utilizes PMUs to transfer information downstream to the distribution network making reactive power provision from distributed assets feasible. The contributions of this paper are listed hereafter:

- Proposal of an efficient way for delivering reactive power across transmission and distribution systems both in a static and dynamic way.

- Utilization of transmission-connected PMUs to increase visibility of system conditions upstream in the network
TABLE I

COMPARATIVE REVIEW OF RECENT SOLUTIONS FOUND IN THE LITERATURE

\begin{tabular}{ll}
\hline Reference & Limitations \\
\hline$[10]$ & $\begin{array}{l}\text { Only PVs are considered. } \\
\text { Changes to controllers are required. }\end{array}$ \\
{$[11]$} & $\begin{array}{l}\text { Only SGs and DFIGs are considered. } \\
\text { Additional controllers at each distribution network are required. }\end{array}$ \\
{$[12]$} & $\begin{array}{l}\text { Specific technologies utilized are not defined. } \\
\text { Studies include static OPF calculations no dynamics are considered. } \\
\text { Specific technologies utilized are not defined. }\end{array}$ \\
{$[13]$} & $\begin{array}{l}\text { Studies include static OPF calculations no dynamics are considered. } \\
\text { Technologies are not well-defined. The description indicates } \\
\text { that resources could be either PVs or energy storage device. } \\
\text { Studies include static OPF calculations no dynamics are considered. }\end{array}$ \\
\hline
\end{tabular}

and support increased resource provision from distributed assets.

- The strategy enables TSO-DSO interaction for increased utilization of the network resources, hence it supports cost-effective transition to low-carbon power systems and reduced asset stranding.

- The proposed strategy takes into account any equipment existing in power systems with reactive power capability. A review of reactive devices is provided to clarify the capability of each device across various timeframes.

- Detailed simulation studies to demonstrate the effectiveness of the proposed approach. The studies extend to phasor-based dynamic stability (PowerFactory DIgSILENT) and electromagnetic transient simulations (PSCAD/EMTDC) to scrutinize the performance of a large-scale transmission-distribution network.

- Hardware-in-the-loop (HIL) simulations to validate the practical feasibility of the concept.

These features enable the framework to reduce the need for investment in dedicated reactive compensating equipment (as also proven by appropriate simulation studies), while addressing voltage abnormalities arising across the system. Overall, the proposed framework leads to an increase of the system flexibility offering to system operators more options at any given time to optimally and securely operate their power systems. These contribution are well-aligned with current developments in the sector highlighting the need for appropriate Volt/Var schemes taking full advantage of distributed reactive power capabilities [15], [16].

\section{Proposed StRategy For PROVISiON OF VOltage ANCILLARY SERVICES}

\section{A. Impact of DGs Reactive Power Exchange on Voltage Sequence Components}

For a three-phase power source, the instantaneous active power $p$ and reactive power $q$ at the PCC can be represented by (1) and (2) respectively (zero sequence components have been neglected) [17].

$$
p=\overbrace{\underbrace{P^{+} \times i_{p}^{+}}_{\text {steady-state component }}+\overbrace{v^{-} \times i_{p}^{-}}^{P^{-}}}^{v_{\text {oscilating component }}^{v^{+} \times i_{p}^{-}+v^{-} \times i_{p}^{+}}}
$$




$$
q=\underbrace{\overbrace{v_{\perp}^{+} \times i_{q}^{+}}^{Q^{+}}+\overbrace{v_{\perp}^{-} \times i_{q}^{-}}^{Q^{-}}}_{\text {steady-state component }}+\underbrace{v_{\perp}^{+} \times i_{q}^{-}+v_{\perp}^{-} \times i_{q}^{+}}_{\text {oscilating component }}
$$

where $v$ is the voltage vector, $i_{p}$ and $i_{q}$ are the active and reactive currents, while superscripts + and - denote the positive and negative sequence components respectively.

Since the average numerical value of the oscillating terms is zero, their impact is neglected from the analysis presented in this paper [18].

Fig. 1 illustrates a simplified representation of a power system as seen from the DGs terminals.

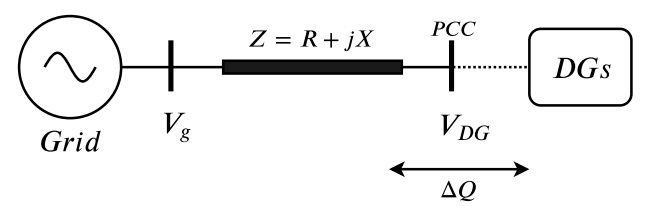

Fig. 1. Simplified representation of a power system as seen from DGs' side.

Considering an equivalent impedance $Z$ between the DGs and terminal $V_{g}$, the voltage $V_{D G}$ at the DG terminal can be expressed by Equation (3).

$$
V_{D G}=V_{g}+R i+L \frac{d i}{d t}
$$

By neglecting the resistive component, expression (3) can be expressed in the $\alpha-\beta$ frame by Equations (4).

$$
\begin{aligned}
& V_{D G \alpha}=V_{g \alpha}+L \frac{d i_{\alpha}}{d t} \\
& V_{D G \beta}=V_{g \beta}+L \frac{d i_{\beta}}{d t}
\end{aligned}
$$

By decomposing the $\alpha-\beta$ frame into symmetrical components, the expressions in (4) can be re-written in Equations (5) and (6).

$$
\begin{aligned}
& \sqrt{3} V_{D G}^{+} \sin \left(\omega t+\phi_{D G}^{+}\right)+\sqrt{3} V_{D G}^{-} \sin \left(\omega t+\phi_{D G}^{-}\right)= \\
& \sqrt{3} V_{g}^{+} \sin \left(\omega t+\phi_{g}^{+}\right)+\sqrt{3} V_{g}^{-} \sin \left(\omega t+\phi_{g}^{-}\right)+ \\
& \omega L \sqrt{3} I^{+} \cos \left(\omega t+\theta^{+}\right)+\omega L \sqrt{3} I^{-} \cos \left(\omega t+\theta^{-}\right) \\
& -\sqrt{3} V_{D G}^{+} \cos \left(\omega t+\phi_{D G}^{+}\right)+\sqrt{3} V_{D G}^{-} \cos \left(\omega t+\phi_{D G}^{-}\right)= \\
& -\sqrt{3} V_{g}^{+} \cos \left(\omega t+\phi_{g}^{+}\right)+\sqrt{3} V_{g}^{-} \cos \left(\omega t+\phi_{g}^{-}\right)+ \\
& \omega L \sqrt{3} I^{+} \sin \left(\omega t+\theta^{+}\right)-\omega L \sqrt{3} I^{-} \sin \left(\omega t+\theta^{-}\right)
\end{aligned}
$$

where $\phi$ and $\theta$ are the angles of voltage and current respectively.

Considering that the objective is provision of voltage support and assuming reactive currents are injected from the DG side towards the PCC, the angle difference difference between the sequence voltage and sequence currents is expressed as below:

$$
\phi^{+}-\theta^{+}=\pi / 2, \quad \phi^{-}-\theta^{-}=-\pi / 2
$$

By adding (5) and (6) into both sides of the equation and amalgamating (7), the following expressions can be derived:

$$
\begin{aligned}
& V_{D G}^{+}=V_{g}^{+}+\omega L I^{+} \\
& V_{D G}^{-}=V_{g}^{-}-\omega L I^{-}
\end{aligned}
$$

Equation (8) indicates that voltage sequence components (either $V_{g}$ or $V_{D G}$ ) are affected by the reactive power components $Q^{+} \& Q^{-}$(refer to Equation (2)). The reactive power component requirements for voltage control are presented in Table II.

TABLE II

SEQUENCE REACTIVE POWER REQUIREMENT FOR VOLTAGE CONTROL.

\begin{tabular}{lc}
\hline Desired effect & Sequence reactive power requirement \\
\hline Increase $V^{+}$ & Inject $Q^{+}\left(Q^{+}>0\right)$ \\
Decrease $V^{+}$ & Absorb $Q^{+}\left(Q^{+}<0\right)$ \\
Increase $V^{-}$ & Absorb $Q\left(Q^{-}<0\right)$ \\
Decrease $V^{-}$ & Inject $Q\left(Q^{-}>0\right)$ \\
\hline
\end{tabular}

\section{B. Control Strategy of DGs and Reactive Power Provision}

Previous work conducted in [19] has been used to formulate the current references of the DGs considering grid voltage $\left(V_{g}\right)$ variations:

$$
\begin{gathered}
i^{+}=k_{1} \frac{P^{*}}{\left|v^{+}\right|^{2}} v^{+}+k_{2} \frac{Q^{*}}{\left|v^{+}\right|^{2}} v_{\perp}^{+} \\
i^{-}=\left(1-k_{1}\right) \frac{P^{*}}{\left|v^{-}\right|^{2}} v^{-}+\left(1-k_{2}\right) \frac{Q^{*}}{\left|v^{-}\right|^{2}} v_{\perp}^{-}
\end{gathered}
$$

where $k_{1}$ and $k_{2}$ are the factors that define and control the proportion between the positive- and the negative-sequence components in the reference currents.

By substituting (9) and (10) into (1) and (2) and neglecting the oscillating components, the instantaneous active power $p$ and reactive power $q$ at the PCC can be re-written as:

$$
\begin{aligned}
& p=\overbrace{k_{1} P^{*}}^{P^{+}}+\overbrace{\left(1-k_{1}\right) P^{*}}^{P^{-}} \\
& q=\overbrace{k_{2} Q^{*}}^{Q^{+}}+\overbrace{\left(1-k_{2}\right) Q^{*}}^{Q^{-}}
\end{aligned}
$$

Equations (2), (8) and (12) indicate that the voltage across the network is determined by the flow of reactive currents, emanating from the corresponding reference points of the associated control strategies.

In the proposed strategy, voltage control is considered and the input signals to the local controller of DGs are the widearea voltage measurements from the remote end of a network (i.e. $V_{g}$ in the example network of Fig. 1).

Considering inverter-based generation, the voltage $V_{g}$ would be a function of reactive power sequence component $q$, which is a function of the reactive currents $i_{q}^{+}$and $i_{q}^{-}$that in turn depend on the upper and lower level control of inverters:

$$
V_{g} \propto q \propto i_{q}^{+}, i_{q}^{-} \propto \underbrace{\left(k_{p} \Delta v+k_{i} \int \Delta v d t\right)}_{\text {PI-based voltage control }}
$$


where $k_{p}$ and $k_{i}$ are the PI gains of the voltage control loop and $\Delta v$ is the error between the voltage reference and the voltage measurement.

Fig. 2 presents a sensitivity analysis of voltage drop $\Delta v$ (as seen by the local voltage controller of DGs) and the reactive current produced. As it can be derived, the reactive current produced by distributed resources when subjected to a local voltage input would be smaller, thus the voltage support more limited. This is why the proposed strategy utilizes voltage measurements from phasor measurement units (PMUs) transferring information from across the system in a way to increase visibility of voltage disturbances and maximize the support provided. This is an effective way of ensuring that DGs provide their maximum reactive power, while keeping the necessary infrastructure intervention to the minimum; note that only one PMU is necessary per transmission-connected substation of interest. Further details on the specific case study investigated in this paper can be found on Section III.

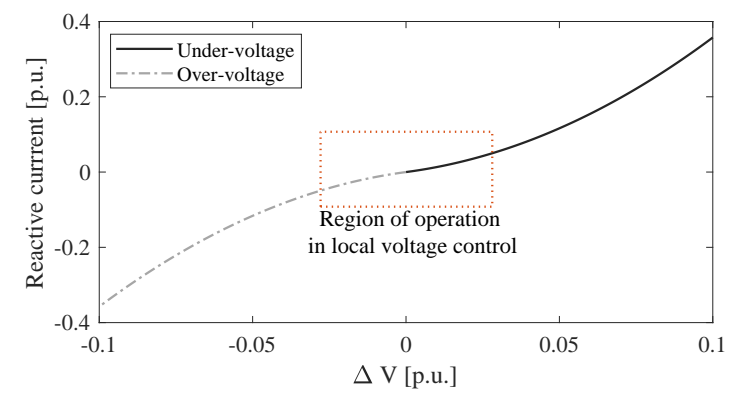

Fig. 2. $\Delta v$ and reactive current sensitivity analysis.

\section{Features of the proposed strategy}

A suitable strategy, as illustrated in Fig. 3, that determines the provision of VAS across transmission and distribution systems is described in this section. The primary feature of the proposed strategy is that it aims to satisfy the needs of the system during operational timescales, as well as to increase the operational flexibility. That is in conflict with the traditional paradigm, where the current frameworks have been designed based on the capabilities of the installed SGs [9]. The current approach leads to system-specific frameworks, which are not beneficial in the era of smart grid, in which increased penetration of non-synchronous generators and other flexible technologies are considered. Another main feature of the proposed strategy is the fact that services are proposed to be unbundled [9]. To further explain this, traditionally SGs sign contracts with system operators for active power provision and provide reactive power to their capacity as part of these contracts. The proposed strategy aims at modifying reactive power flows from the various equipment irrespective of the active power provision to the system; again this can increase the system flexibility. Of course, the latter raises concerns with regards to the compensation of such unbundled services. The idea behind the proposed strategy would be for an appropriate market mechanism to be established and accordingly compensate the participated equipment. In conflict with this, voltage services are mostly uncompensated at the moment.

\section{Elements that define the strategy}

Three elements are defined towards the design of the strategy [7], [8]:

- AS target. This element defines the purpose, for which the service is required. The AS target highly relates to the voltage management requirements imposed by system operators; e.g. voltage regulation during steady-state and voltage containment and recovery processes during and after a disturbance. For this paper, this can be the need of the system to (i) maintain steady voltages, (ii) control the voltage during disturbances, and (iii) maintain a sufficient voltage stability margin ensuring a stable power system under a credible set of contingencies.

- AS outcome. This element defines a technical set of specifications, which highlight the content of the service. In all three objectives mentioned above, the product is the provision of reactive power from the involved equipment. Two different VAS are identified, (i) Static Voltage Support and (ii) Dynamic Voltage Support. The two services differ in the timeframe within which reactive power is supplied, and also the problem they are resolving. Static voltage support would be required for controlling voltages across the system and the efficient transmission of active power, therefore it should be provided in a relatively slow timescale. Dynamic voltage support would be required for mitigating the issue of decreasing system strength, which becomes topical in cases of high penetration of non-synchronous technologies, hence alleviate instabilities during voltage disturbances.

- AS procurement. The process of an AS product from the user to the system operator. For example, through a contract. This paper will not deal with this, however various market models are discussed in [7].

\section{E. Equipment considered in the proposed strategy}

This section provides an analysis of the features of the equipment considered in the strategy [20], [21]. Table III highlights the most appropriate of the equipment for provision of reactive power. A ranking of the equipment is revealed based on three criteria, which we consider of utmost importance for categorizing the involved equipment and assessing its potential for provision of VAS. The criteria are:

1) Timeframe for undertaking action. The equipment analyzed operates in different timescales ranging from milliseconds (e.g. inverter-interfaced generators) to seconds (transformer tap changers).

2) Operational capability. This is defined as either continuous in a certain range (e.g. PQ capability curve of SGs) or fixed with discrete values (e.g. discrete steps typically characterizing capacitor banks).

3) Need for investment. Some of the equipment (e.g. SGs, DG units, OWFs, etc.) is used, in principle, for providing active power, however they have an inherent capability 




Fig. 3. Outline of the proposed strategy illustrating DCRs and TSO-DSO interface.

for reactive power support. This equipment is considered not to require additional investment for installation. On the other hand, some of the proposed equipment is used purely for reactive power support (e.g. capacitors banks, STATCOMs, etc.), hence it is coupled with an investment cost required for bringing it online.

TABLE III

ASSESSMENT OF VOLTAGE CONTROL EQUIPMENT

\begin{tabular}{lllll}
\hline \hline $\begin{array}{l}\text { Equipment } \\
\text { type }\end{array}$ & Timeframe & Capability & $\begin{array}{l}\text { Investment } \\
\text { required? }\end{array}$ & Ranking \\
\hline DG units & $\mathrm{ms}$ & Continuous & No & 1 \\
\hline OWF & $\mathrm{ms}$ & Continuous & No & 2 \\
\hline $\begin{array}{l}\text { VSC-HVDC } \\
\text { links }\end{array}$ & $\mathrm{ms}$ & Continuous & No & 3 \\
\hline $\begin{array}{l}\text { Synchronous } \\
\text { generators }\end{array}$ & $\mathrm{ms}$ & Continuous & No & 4 \\
\hline $\begin{array}{l}\text { Synchronous } \\
\text { condensers }\end{array}$ & $\mathrm{ms}$ & Continuous & Yes & 5 \\
\hline STATCOMs & $\mathrm{ms}$ & Continuous & Yes & 6 \\
\hline uPFC & $\mathrm{ms}$ & Continuous & Yes & 7 \\
\hline $\begin{array}{l}\text { Energy } \\
\text { storage }\end{array}$ & $\mathrm{ms}$ & Continuous & No & 8 \\
\hline SVCs & $\mathrm{ms}$ & Fixed & Yes & 9 \\
\hline $\begin{array}{l}\text { Tap stagger } \\
\text { operation }\end{array}$ & $\mathrm{sec}$ & Fixed & No & 10 \\
\hline $\begin{array}{l}\text { Capacitors } \\
\& \text { reactors }\end{array}$ & $\mathrm{sec}$ & Fixed & Yes & 11 \\
\hline $\begin{array}{l}\text { Tap changer } \\
\text { operation }\end{array}$ & $\mathrm{sec}$ & Fixed & No & 12 \\
\hline \hline
\end{tabular}

With respect to the given ranking, it is firstly noted that the ranking tries to capture a future power system setup, where much of generation is distributed across the system. Secondly, it is necessary to be noted that this is not a onefit-for-all ranking and depending on the power system under investigation and the availability of the equipment each system operator shall decide what would be best for each case. As such, the ranking given encapsulates a situation specific to the GB power system and draws from the authors' knowledge of the particularities of this power system and the choices made by the TSOs and DNOs.

The equipment is divided based on their capability to offer reactive power dynamically or in a static way. All equipment offering reactive power in a static way are automatically ranked in the bottom of the list; i.e. tap stagger operation, tap changers and capacitors and reactors are in the last three places of the ranking. Of course, implementation of tap stagger operation and tap changers are quite inexpensive and that is why they are assumed to be higher than capacitors and reactors, which come with an upfront cost. A further reason for these devices to be ranked in the bottom of the list is inability to modify their output on a continuous range based on a reference measurement. This indicates why all equipment with dynamic capability for VAS service provision are in the first nine places in the list (refer to DG units down to SVCs).

Considering capital cost to be the second criterion for the ranking, we start prioritizing equipment requiring insignificant capital cost for the provision of VAS higher in the list. This explains why DG units and offshore wind are placed in the top of the list, since it is assumed that the units exist in the system and would require only measurement devices, communications links and relevant control modification to offer a VAS (i.e. much cheaper than installing reactive power compensating equipment). Then, VSC-HVDC links are ranked third, as relevant literature has shown that a transition to RES penetration levels close to $100 \%$ would require vast amount of interconnection between countries and continents [22]. Considering that these links would probably be MMCbased, as is the state-of-the-art these days, they would have capability to operate even in STATCOM mode, which would offer them great capabilities for reactive power provision. Moving down the list, synchronous generators and condensers essentially have the same capabilities for provision of reactive 
power in a dynamic way. It is highlighted here that the only reason for not ranking these two devices higher relates to the fact that this paper captures a future paradigm, in which transmission-connected synchronous generators (and therefore condensers) become more scarce; note the plans that several governments across the planet have introduced for decommissioning conventional power plants (i.e. based on synchronous generator technology) [23], [24], [25]. STATCOMs and uPFCs come straight after, as they arguably have great capabilities for fast responses in a dynamic way, with their only downside being the high upfront cost required to bring them online. Energy storage follows in the ranking, as storage devices have control capabilities allowing them to dynamically provide reactive power. However, energy storage is currently at the higher end of costly devices. SVCs are the last device with dynamic capabilities, as they require upfront cost and are typically characterized by slower responses than other devices higher in the list (e.g. STATCOMs).

\section{F. Actors' role towards realizing the strategy}

The utilization of the above equipment for managing voltages across transmission-distribution systems and mitigating instability issues suggests a different system management approach [7]. The participated entities may need to adjust their role in the system operation to enable the services to be exchanged across the systems. Table IV shows how the role of the involved participants could deviate from the businessas-usual approach.

TABLE IV

ACTORS PARTICIPATING IN THE STRATEGY

\begin{tabular}{cll}
\hline \hline Actor & $\begin{array}{l}\text { Business-as-usual } \\
\text { responsibilities }\end{array}$ & Additional responsibilities \\
\hline TSO & $\begin{array}{l}\text { Operate system, address } \\
\text { transmission constraints }\end{array}$ & $\begin{array}{l}\text { Utilization of reactive response } \\
\text { from distribution system }\end{array}$ \\
\hline DSO & $\begin{array}{l}\text { System operator, address } \\
\text { distribution constraints }\end{array}$ & $\begin{array}{l}\text { Utilization of reactive response from } \\
\text { DGs, flexibility to TSO for resolving } \\
\text { transmission constraints }\end{array}$ \\
\hline Distributed resources & $\begin{array}{l}\text { Passive role, mainly } \\
\text { exporting active power } \\
\text { under contracts }\end{array}$ & $\begin{array}{l}\text { Active role by offering reactive } \\
\text { support for resolving transmission } \\
\text { and distribution constraints }\end{array}$ \\
\hline \hline
\end{tabular}

For such an approach to be realized, significant changes need to be implemented especially relating to information exchange between the interested parties; i.e. additional data need to be shared, primarily, in the interface between TSOs and DSOs. A recently formed Joint Working Group among CIGRE and CIRED (CIGRE/CIRED JWG C1.29 [26]) has reviewed planning criteria across the world. The review identified the information that needs to be exchanged leading to enhanced collaboration between the two entities, in order for the system security to be maximized and the investment minimized in the presence of high DG penetration. An outcome of the Working Group suggests that TSOs do not generally have direct monitoring of DG units, and no direct control over them (in most countries they need to instruct the DSO to control them if necessary). As such, it is recommended that the above actions shall be implemented in an automatic fashion indicating the need for innovative control systems coupled with appropriate ICT technologies to enable cross-communication; the strategy proposed in this paper is in line with this. For the proposed strategy to be implemented, DSOs should provide to TSOs information on active and reactive power capabilities of their plants in realtime, along with the types of generators (and vice versa for TSOs). This should be taken into account not only in planning models (as suggested by the Working Group) but also in real-time operational dispatch models. The same principle should also apply to DSOs who should receive real-time information about the condition of the transmission system, so that they best operate their assets for supporting the system in case of large disturbances. For example, the transmission of time-synchronized voltage measurements (using PMUs) from transmission system grid supply points (GSPs) to DG units would be useful in improving the support of the distribution network to the transmission system [27]. This is showcased in Section III.

\section{Simulations FOR DEMONSTRATING THE EFFECTIVENESS OF THE STRATEGY}

This section includes a selection of studies to demonstrate the applicability and benefits of the strategy. The network topology has been selected as it reflects a highly decentralized power system anticipated in the near future, hence it is used as a benchmark network for studying voltage stability issues in-depth and the provision of VAS for their mitigation. The network, as shown in Fig. 4, is a highly meshed one comprising of a transmission system connected to several distribution systems. It includes PV plants, onshore and offshore wind farms, HVDC links and transformers fitted with tap stagger (TS) operation. In addition to these, STATCOMs have been incorporated in the model to ensure that a significant number of the equipment discussed in Table III are considered in these studies. This complex network is utilized for the dynamic phasor-domain simulations (refer to subsection III-A). For practical purposes, the distribution system downstream $\mathrm{GSP}_{2}$ is the one adopted for the transient simulations and real-time hardware validation (refer to subsections III-B and IV-A).

\section{A. Dynamic phasor-based simulations}

For the RMS simulations, the detailed transmissiondistribution network of Fig. 4 comprising of several hundreds transmission-level $(400 / 275 \mathrm{kV})$ substations and several distribution networks $(132 / 33 \mathrm{kV})$, has been developed in PowerFactory DIgSILENT. The utilized network includes a significant penetration of renewable energy sources connected at all voltage levels. OWFs and VSC-HVDC links with a total capacity of $1 \mathrm{GW}(2 \times 500 \mathrm{MW})$ and $2 \mathrm{GW}(2 \times$ $1 \mathrm{GW})$ respectively are considered. Additionally, the pairs of transformers connecting the transmission system to the distribution have been set up to provide TS operation during overvoltages [28], [29]. All network components include detailed dynamic models and represent specific schemes; solar PV plants and wind farms have been modeled as per [30] and [31] respectively, while the control of VSC-HVDC links has been based on the control strategy proposed in [32].

The following subsections illustrate the performance of the utilized network when a solid three-phase fault is applied to 


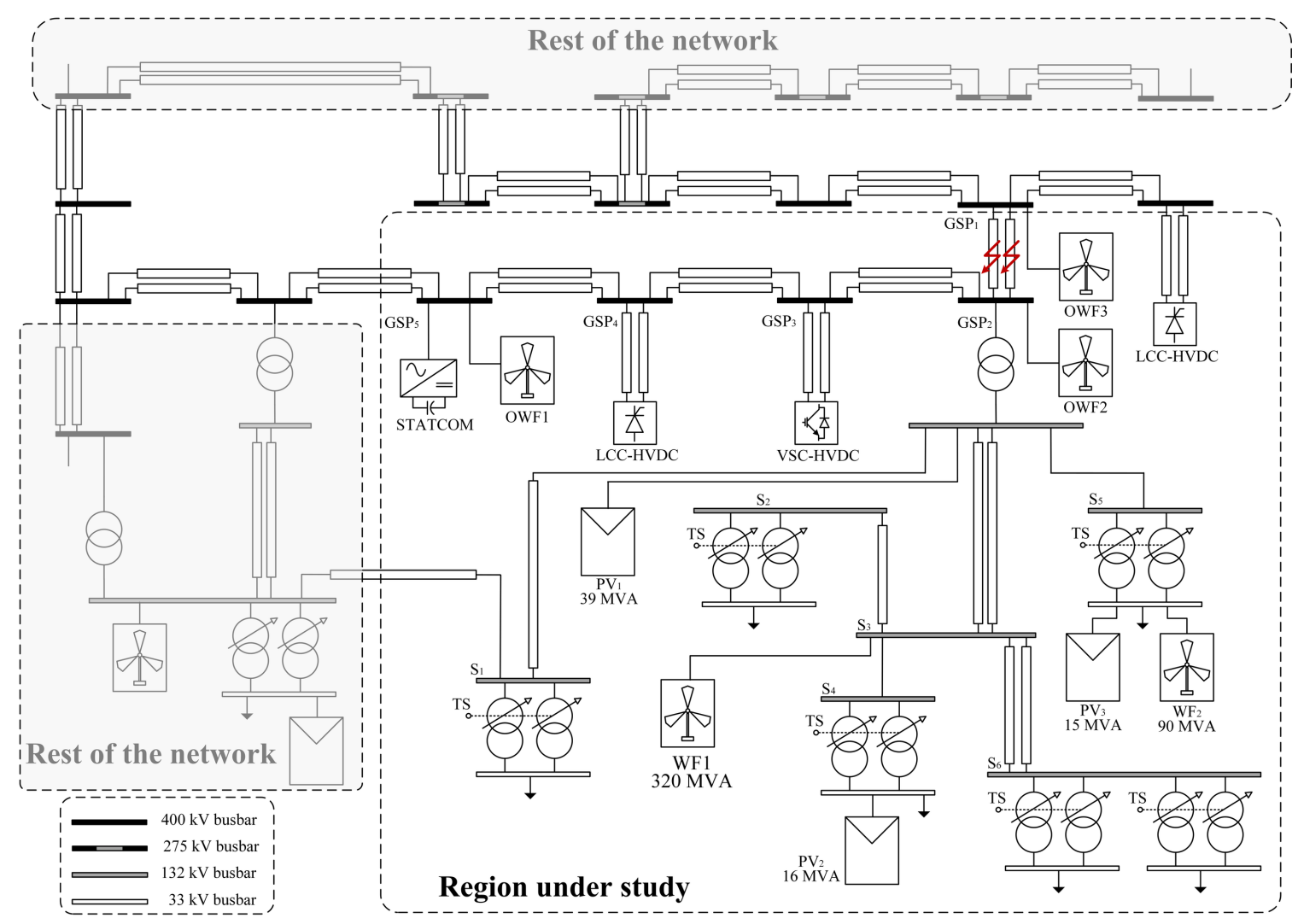

Fig. 4. Schematic of network under consideration.

the lines connecting $\mathrm{GSP}_{1}$ and $\mathrm{GSP}_{2}$. The fault is cleared after $100 \mathrm{~ms}$ through circuit breaker operation. Two sets of operating conditions are investigated:

- Scenario I - Minimum system loading, high renewable generation, and HVDC links out-of-service.

This operating condition results in overvoltages. Fig. 5 shows the resulting overvoltages. Minor overvoltages above the allowed limit are observed in four GSPs (i.e. $\mathrm{GSP}_{2}, \mathrm{GSP}_{3}$, $\mathrm{GSP}_{4}, \mathrm{GSP}_{5}$ ).

- Scenario II - Maximum system loading, high renewable generation, and HVDC links in export operation.

Fig. 6 illustrates the voltage profiles of the various GSPs post-fault. It can be observed that all GSPs, but $\mathrm{GSP}_{1}$, return to abnormal voltage values (i.e. outside the typically admissible range of 0.95 - 1.05 p.u.). $\mathrm{GSP}_{2}$ is the most severely affected substation, hence it is brought forward in the following sections to showcase the benefit of absorbing reactive power from the installed network equipment. Note that the legend appended in Fig. 5 indicates the average value of the voltage profile post-fault and specifically calculated from $1 \mathrm{sec}$ to $2 \mathrm{sec}$, when the system has reached its steadystate condition; the same applies to the rest of the figures presenting voltage profiles within this paper.

1) Comparative study to show the benefit of PMUs: As mentioned in Section II-F, PMU devices can prove to be very useful in sharing information across the various system levels. In this section, a PMU data stream collected from $\mathrm{GSP}_{2}$ is transferred to all DG units that are requested to support the

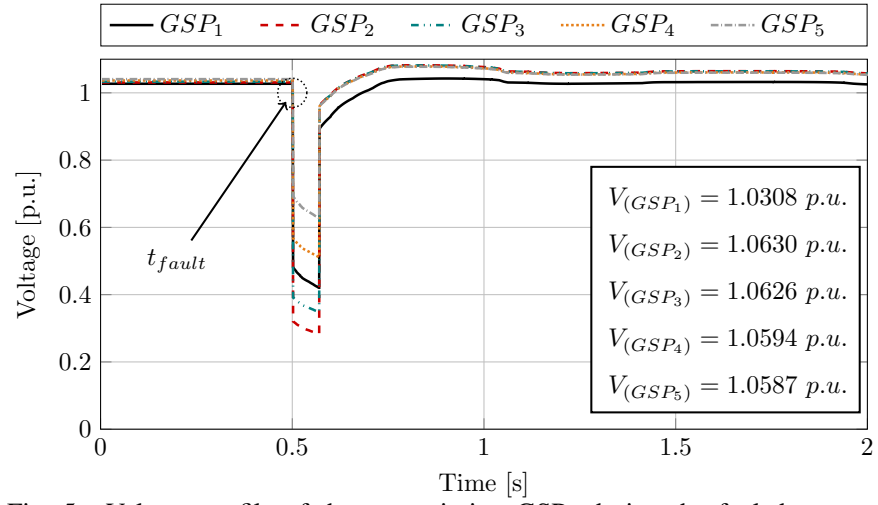

Fig. 5. Voltage profile of the transmission GSPs during the fault between $\mathrm{GSP}_{1}$ and $\mathrm{GSP}_{2}$ in Scenario I.

system during a solid three-phase fault in the line connecting $\mathrm{GSP}_{1}$ and $\mathrm{GSP}_{2}$. Fig. 7 illustrates the difference in reactive power generation from three onshore wind farms (i.e. $W F_{1}$ from Fig. 4 and two more connected in the distribution system of $\mathrm{GSP}_{3}$ ) with a total capacity of 700 MVA with and without utilization of PMU measurements.

It is noted that for all studies conducted below, DG units and offshore wind farms are fitted with PMU data streams in order to increase their visibility over transmission system contingencies.

2) Dynamic Voltage Support: This section seeks to demonstrate the benefit of the proposed dynamic voltage service through a comparative study with STATCOMs. The choice 




Fig. 6. Voltage profile of the transmission GSPs during the fault between $\mathrm{GSP}_{1}$ and $\mathrm{GSP}_{2}$ in Scenario II.



Fig. 7. Reactive power generation from onshore WFs during a fault with and without PMU measurements.

for comparison with STATCOM devices is based on the fact that there has recently been a clear trend from TSOs to invest in reactive power compensating devices for ensuring secure operation under such contingencies [5]. Fig. 8 and Fig. 9 illustrate the mitigation of the resulting voltage deviation from the nominal value during the two scenarios. Especially with reference to the undervoltage scenario (refer to Fig. 9), which is the most severe case, it is observed that STATCOMs can marginally boost the voltage up to the acceptable value of 0.95 pu. Note that 4 x 200 MVAr STATCOMs have been placed at transmission level, one at each of the GSPs. The combined reactive power generation from DG units, OWFs and VSCHVDC links achieves improved post-fault recovery of up to 0.97 p.u.

Effective performance can also be observed in the overvoltage case (Fig. 8), where the slight overvoltage is mitigated through the absorption of several hundreds of MVArs. Note that for this study TS operation of the transfomer pairs found in the system (i.e. $34 \mathrm{x}$ of $132 / 33 \mathrm{kV}$ pairs) has been enabled. It is worth pointing out here that a delay of $300 \mathrm{~ms}$ has been imposed in the tap stagger operation to account for the slow nature of this equipment. This, in conjunction with their limited availability to absorb reactive power (i.e. approximately $38 \mathrm{MVAr}$ in overall), suggests that they could be used in cases of slight overvoltages like the one investigated here. However, they would not have the capability to support the system in cases of higher stress.
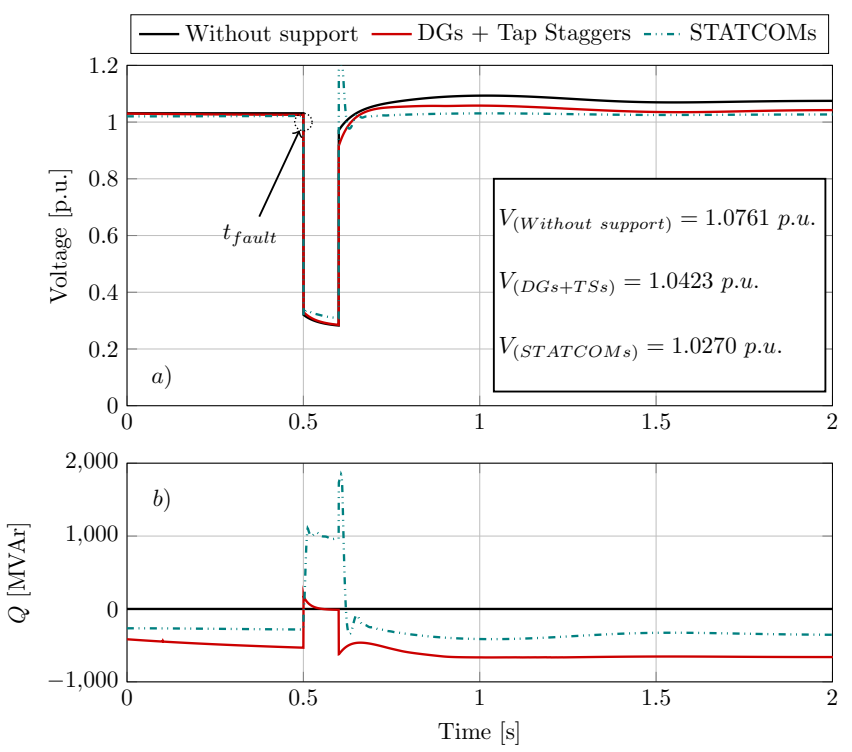

Fig. 8. System response in Scenario I with support from various system resources: (a) voltage profile of $\mathrm{GSP}_{2}$, (b) reactive power absorption.
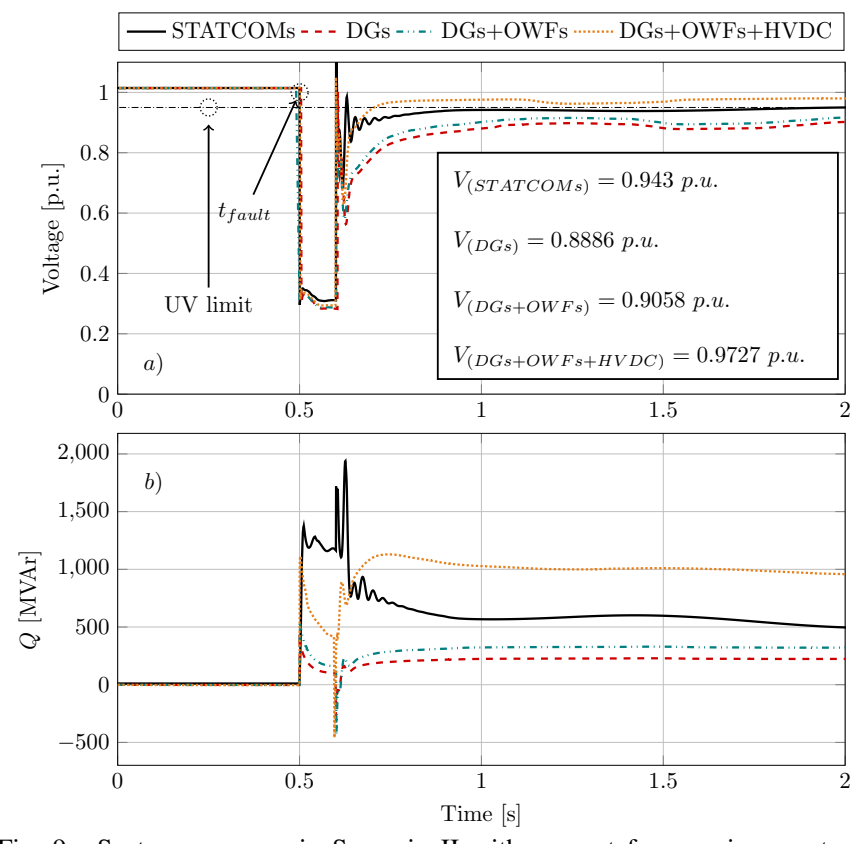

Fig. 9. System response in Scenario II with support from various system resources: (a) voltage profile of $\mathrm{GSP}_{2}$, (b) reactive power generation.

To allow a broader set of resources to be investigated, as per Table III, one more case study is explored relating to Scenario II. It is assumed that an 800 MVA synchronous condenser and an $800 \mathrm{MW} / 2,400 \mathrm{MWh}$ battery energy storage system (BESS) have been installed in $G_{S P} P_{3}$. Note that the devices have been installed in $G S P_{3}$ to increase the electrical distance from the fault, while only one of each device has been installed to allow for an illustrative case (hence their rated capacity is equal to the overall capacity of the installed STATCOMs). The synchronous condenser is modeled as a standard salient-pole synchronous machine [33]. Three control systems have been integrated which include i) automatic voltage regulator (AVR), ii) power system stabi- 
lizer and iii) over-excitation limiter. The prime mover and governor have been omitted as well as there is no mechanical input. The BESS is a state-of-the-art lithium ion battery to allow for fast response, which has been based on a $13 \mathrm{Ah}$ cells with the ratings of $10 \mathrm{MW}, 3.34 \mathrm{MWh}, 1.3035 \mathrm{kV}, 3 \mathrm{C}$ charging/discharging current.



Fig. 10. Reactive power benchmarking for STATCOMs, synchronous condenser and BESS.

Fig. 10 achieves the characterization of the response of the two devices, and essentially demonstrates a similar response to the one by the STATCOMs. It is observed that post-fault (i.e. mainly investigated in this paper) the STATCOMs are more aggressively tuned, while the synchronous condenser is subject to AVR and physical dynamics owning its slower response. The control frame of the BESS is a typical $d-q$ current injection control system subject to droop setting and PI tuning.

3) Static Voltage Support: To demonstrate that reactive power resources can supplement the ability of the system to withstand voltage variation during normal operation and the onerous condition identified in the previous sections (i.e. line outage between $\mathrm{GSP}_{1}$ and $\mathrm{GSP}_{2}$ ), P-V curves are employed in this section. Suitable P-V curves of the network under investigation are being drawn to demonstrate how the loss of the line between the two GSPs can impact on the region's loadability limit. Fig. 11 shows the loadability limit of the region before any disturbance (i.e. 5.5 $\mathrm{GW}$ ) and after the outage (i.e. $3.7 \mathrm{GW}$ ). Minimum and maximum load of this network is $2.4 \mathrm{GW}$ and $4.2 \mathrm{GW}$ respectively. As such, it can be observed that during peak demand the line outage would lead to undesirable load shedding.

DG units and connected OWFs in the vicinity of the region are deployed to provide reactive power and increase its loadability limit even during the investigated outage. Fig. 11 illustrates that with DG units supplying $220 \mathrm{MVAr}$ and OWFs supplying $340 \mathrm{MVAr}$, the limit is increased to $4.1 \mathrm{GW}$ and $4.3 \mathrm{GW}$ respectively, hence covering the peak demand. It is worth pointing out that OWFs are not as effective as the DG units and this relates to the fact that DG units supply reactive power in a dispersed manner covering reactive power shortage across the system in a more effective way.

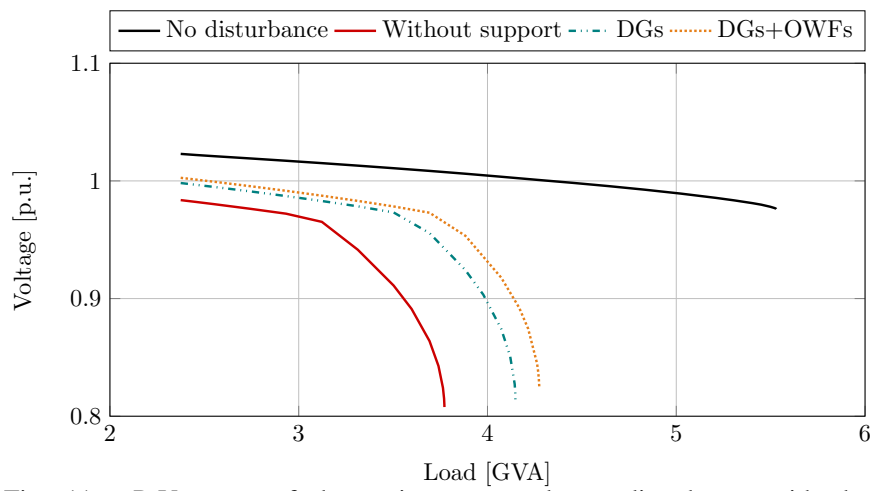

Fig. 11. P-V curve of the region pre- and post-disturbance with the deployment of various reactive power resources.

\section{B. Electromagnetic transient simulations}

To conduct EMT-based simulations, the distribution system downstream $\mathrm{GSP}_{2}$ has been developed in PSCAD/EMTDC to accurately capture the transient characteristics of the system. Since Scenario II is more severe, it is selected here as the case study. Fig. 12(a) illustrates the voltage upon a fault on the busbar of $\mathrm{GSP}_{2}$. The installed DG units are deployed to provide reactive power and reduce the voltage deviation from the nominal value (i.e. 1 p.u.). Fig. 12(b) captures the generation of 300 MVAr reactive power to meet the target voltage value.
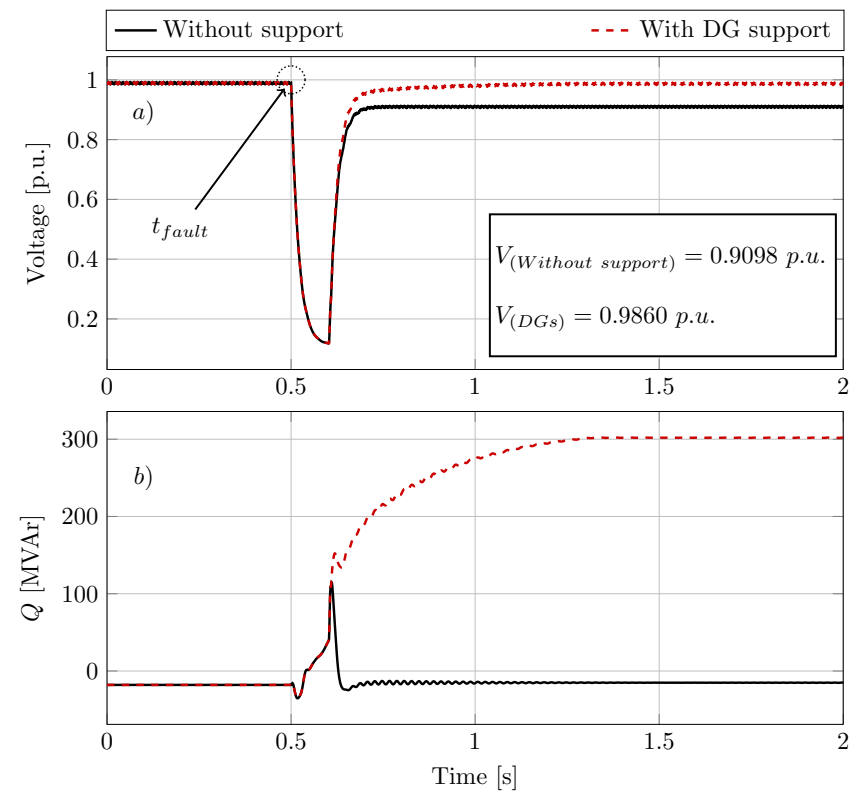

Fig. 12. Scenario II implemented in PSCAD/EMTDC with and without support from DG units: (a) voltage profile of $\mathrm{GSP}_{2}$, (b) reactive power generation of DG units.

\section{EXPERIMENTAL VALIDATION AND DATA FLOW ARCHITECTURES}

\section{A. Real-time hardware-in-the-loop validation}

In order to prove the practical feasibility of the proposed strategy, a real-time hardware prototype has been developed. As depicted in Fig. 13, the entire HIL testbed comprises of 


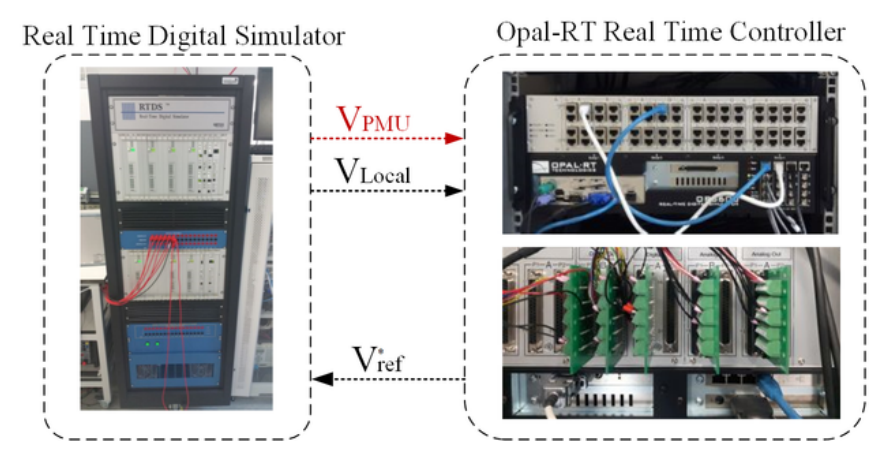

Fig. 13. Experimental setup.

two different sub-systems. The first one has been developed using Real Time Digital Simulator (RTDS) and includes the actual power network (i.e. distribution system downstream $\mathrm{GSP}_{2}$ of Fig. 4). The second sub-system (developed in Opal-RT control prototyping platform) is an actual controller required for the implementation of the proposed strategy. The input signals to the controller are the wide-area voltage measurements from the transmission network (i.e. $V_{P M U}$ ) and the local measurements from DGs terminals (i.e. $V_{\text {Local }}$ ). The output of the controller is a voltage reference $V_{r e f}^{*}$ signal fed to the local voltage control loops of the corresponding DGs. The HIL simulation results are illustrated in Fig. 14, where sub-figures (a) and (b) indicate the voltage profile of $\mathrm{GSP}_{2}$ during Scenario II, and the overall reactive power generation from DG units installed in the respective distribution system respectively. It can be observed that no support is provided from the DG units when they monitor the voltage at the point of connection (i.e. $V_{\text {Local }}$ ), hence the voltage is severely affected and cannot return to admissible levels post-fault. On the right side, Fig. 14(c) depicts the voltage when PMU measurements are transferred to the DG units; it is shown that the voltage returns to values close to 1 p.u.. For this to happen, Fig. 14(d) indicates that generation of approximately 150 MVAr is necessary (base power used 300 MVA). It shall also be highlighted that no significant delays arising from communication infrastructure have been observed during the experimental validation; please refer to Section IV-B for further details.

\section{B. Data flow architectures for the proposed strategy}

Fig. 15 illustrates the data flow architecture of the proposed strategy. The PMU data from the substation of interest, is sent out in C37.118 format data frame (i.e. the standard mostly used in practice for PMU-based applications). These data are then received (through wide communication networks) at the location of the reactive power resources for utilization within their voltage control devices. It shall be highlighted here that, in this way, only one signal (i.e. voltage at the GSP under investigation) should be transferred. This directly and totally eliminates any delays with respect to multiplexing and queuing delays (even though these could also be eliminated by prioritizing the corresponding data frames).

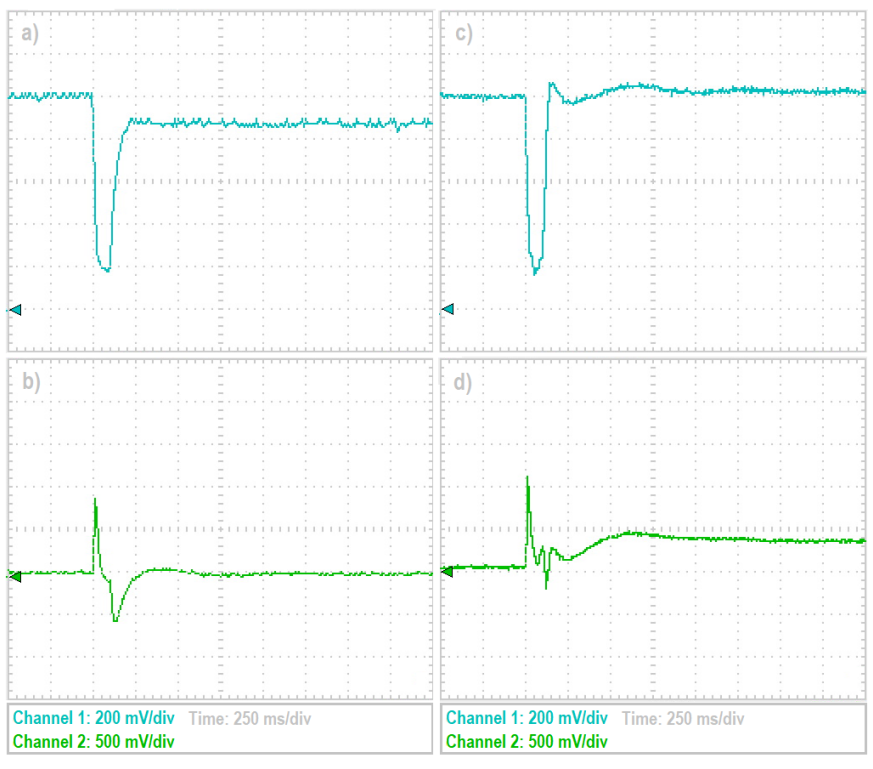

Fig. 14. Scenario II implemented in RTDS/Opal-RT with and without reactive power support: (a) voltage profile of $\mathrm{GSP}_{2}$ without support, (b) reactive power generation with support disabled, (c) voltage profile of $\mathrm{GSP}_{2}$ with support, (d) reactive power generation with support enabled.

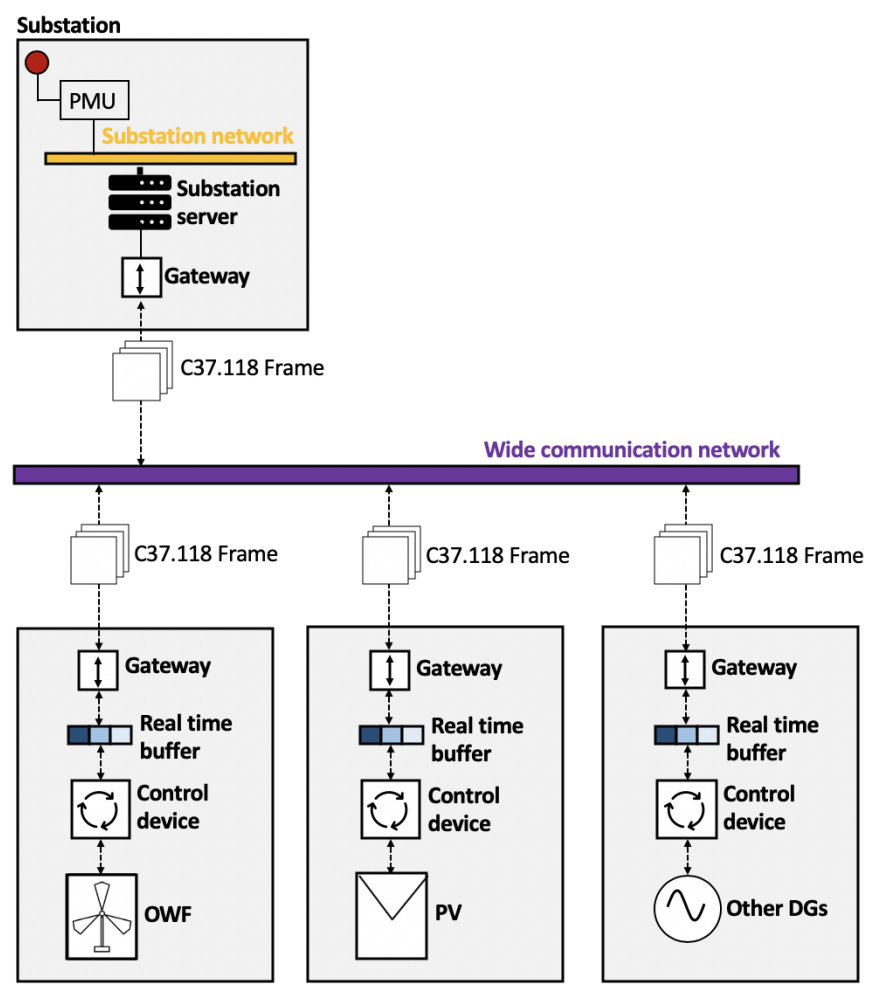

Fig. 15. Data flow architecture of the proposed strategy.

Building on the above, the single-to-be-transferred signal is a phasor (i.e. RMS) which is pre-calculated from time-domain signatures and pre-time stamped at the PMU location. This essentially reduces the data to be transferred (i.e. only an RMS value for every electric cycle need to be transferred rather than a full, high resolution, sine wave) and also diminishes 
the processing requirements.

With respect to the bandwidth and the communication delays, previous work conducted in [34], [35] has indicated that for large data flow exchanges with many devices and increased network traffic, the processing delays can be as low as a few ns, while the communication delays can be on average $30 \mathrm{~ms}$ (considering a P-Class PMU). Considering the time frames of the proposed voltage strategy both processing and communication would have no significant impact.

\section{Conclusions}

This paper has proposed a strategy for provision of VAS in a technology-neutral manner. The system operability needs are placed in the center of this strategy; the primary goal is to ensure a safe and secure power system with respect to voltage stability. For this reason, two ancillary services have been identified, (i) static voltage support and (ii) dynamic voltage support. The two services are deployed when required to ensure that the voltage stability margins are not violated and the voltage deviation does not exceed the admissible levels. Reactive power generation (accordingly absorption) is achieved through suitable voltage control of the various equipment in order to increase (accordingly decrease) the voltage following a disturbance. Extensive simulation studies spanning across dynamic phasor-domain, EMT-type and realtime hardware validation have been conducted to demonstrate the effectiveness of the proposed strategy. Additional aspects with respect to data architectures and data exchange, required for the practical implementation of the proposed strategy, have also been discussed in the paper. It has been proven that the various equipment considered for the strategy can, to a certain extent, support the system in relation to the given need arising in the presence of various operating conditions. Especially given the increased penetration of renewable energy sources both in the form of transmission- and distribution-connected plants, it has been demonstrated that they can provide an alternative to typical reactive power compensating devices (e.g. STATCOMs) in a way to reduce investment costs. In overall, consideration of equipment with some given reactive power capability leads to more options, therefore the flexibility of the system for response during voltage disturbances would be increased.

\section{REFERENCES}

[1] T. Hess, P. Schegner, and M. Schmidt, "Studies on provision of ancillary services by distributed generation units and storage devices," in 2016 IEEE PES Innovative Smart Grid Technologies Conference Europe (ISGT-Europe), Oct 2016, pp. 1-6.

[2] P. Cuffe, P. Smith, and A. Keane, "Transmission system impact of wind energy harvesting networks," IEEE Transactions on Sustainable Energy, vol. 3, no. 4, pp. 643-651, Oct 2012.

[3] D. Pudjianto, C. Ramsay, and G. Strbac, "Virtual power plant and system integration of distributed energy resources," IET Renewable Power Generation, vol. 1, no. 1, pp. 10-16, March 2007.

[4] G. Valverde and T. V. Cutsem, "Control of dispersed generation to regulate distribution and support transmission voltages," in 2013 IEEE Grenoble Conference, June 2013, pp. 1-6.

[5] C. G. Kaloudas, L. F. Ochoa, B. Marshall, S. Majithia, and I. Fletcher, "Assessing the future trends of reactive power demand of distribution networks," IEEE Transactions on Power Systems, vol. 32, no. 6, pp. 4278-4288, Nov 2017.
[6] A. Oulis Rousis, Y. Pipelzadeh, T. Green, and G. Strbac, "Benefits of distributed power generation for voltage support in GB transmission system: Case study on the south-east region," 2017 IET International Conference on Resilience of Transmission and Distribution Networks (RTDN), 2017.

[7] H. Gerard, E. I. R. Puente, and D. Six, "Coordination between transmission and distribution system operators in the electricity sector: A conceptual framework," Utilities Policy, 2017.

[8] S. Glismann and F. Nobel, "A framework for ancillary services design," in 2017 14th International Conference on the European Energy Market (EEM), June 2017, pp. 1-6.

[9] J. Matevosyan, S. Sharma, S. H. Huang, D. Woodfin, K. Ragsdale, S. Moorty, P. Wattles, and W. Li, "Proposed future ancillary services in electric reliability council of texas," in 2015 IEEE Eindhoven PowerTech, June 2015, pp. 1-6.

[10] G. Valverde, D. Shchetinin, and G. Hug-Glanzmann, "Coordination of distributed reactive power sources for voltage support of transmission networks," IEEE Transactions on Sustainable Energy, vol. 10, no. 3, pp. 1544-1553, 2019.

[11] P. Aristidou, G. Valverde, and T. Van Cutsem, "Contribution of distribution network control to voltage stability: A case study," IEEE Transactions on Smart Grid, vol. 8, no. 1, pp. 106-116, 2017.

[12] Z. Li, Q. Guo, H. Sun, and J. Wang, "Coordinated transmission and distribution ac optimal power flow," IEEE Transactions on Smart Grid, vol. 9, no. 2, pp. 1228-1240, 2018.

[13] S. Karagiannopoulos, C. Mylonas, P. Aristidou, and G. Hug, "Active distribution grids providing voltage support: The swiss case," IEEE Transactions on Smart Grid, pp. 1-1, 2020.

[14] D. B. Arnold, M. D. Sankur, M. Negrete-Pincetic, and D. S. Callaway, "Model-free optimal coordination of distributed energy resources for provisioning transmission-level services," IEEE Transactions on Power Systems, vol. 33, no. 1, pp. 817-828, 2018.

[15] K. E. Antoniadou-Plytaria, I. N. Kouveliotis-Lysikatos, P. S. Georgilakis, and N. D. Hatziargyriou, "Distributed and decentralized voltage control of smart distribution networks: Models, methods, and future research," IEEE Transactions on Smart Grid, vol. 8, no. 6, pp. 2999 3008, 2017.

[16] "IEEE standard for interconnection and interoperability of distributed energy resources with associated electric power systems interfaces," IEEE Std 1547-2018 (Revision of IEEE Std 1547-2003), pp. 1-138, 2018.

[17] The Instantaneous Power Theory. John Wiley \& Sons, Ltd, 2017, ch. 3, pp. 37-109.

[18] A. Montanari, Enhanced Instantaneous Power Theory for Control of Grid Connected Voltage Sourced Converters under Unbalanced Conditions, PhD Thesis, 2017.

[19] R. Teodorescu, M. Liserre, and P. Rodrguez, Grid Converters For Photovoltaic And Wind Power Systems. IEEE PRESS - Wiley, 2011.

[20] M. Peikherfeh, M. Abapour, M. P. Moghaddam, and A. Namdari, "Optimal allocation of facts devices for provision of voltage control ancillary services," in 2010 7th International Conference on the European Energy Market, June 2010, pp. 1-5.

[21] B. Kirby and E. Hirst, Ancillary service details: Voltage control, Dec 1997.

[22] G. Strbac, M. Aunedi, D. Pudjianto, D. Sanders, A. Hart, M. Ravishankar, and J. Brunert, "An analysis of electricity system flexibility for great britain," 112016.

[23] P. Markewitz, M. Robinius, and D. Stolten, "The future of fossil fired power plants in germanya lifetime analysis," Energies, vol. 11, p. 1616, 062018.

[24] The Guardian, "UK to be left with five coal power stations after latest closure," 2019, [Online; accessed 28-March-2020]. [Online]. Available: \url\{https://www.theguardian.com/environment/2019/jun/ 13/mild-but-windy-winter-was-greenest-ever-for-uk-energy-use

[25] B. Knop, M. Pahle, H. Kondziella, F. Joas, O. Edenhofer, and T. Bruckner, "Germanys nuclear phase-out: Sensitivities and impacts on electricity prices and co2 emissions," Economics of Energy \& Environmental Policy, vol. 3, no. 1, pp. 89-106, 2014.

[26] CIGRE-CIRED Joint Working Group C1.29, Planning criteria for future transmission networks in the presence of a greater variability of power exchange with distribution systems, Mar 2017.

[27] C. D. Vournas, C. Lambrou, and P. Mandoulidis, "Voltage stability monitoring from a transmission bus pmu," IEEE Transactions on Power Systems, vol. 32, no. 4, pp. 3266-3274, July 2017. 
[28] L. Chen, H. Y. Li, S. Cox, and K. Bailey, "Ancillary service for transmission systems by tap stagger operation in distribution networks," IEEE Transactions on Power Delivery, vol. 31, no. 4, pp. 1701-1709, Aug 2016.

[29] A. Oulis Rousis, Chairiman, Y. Pipelzadeh, T. Green, and G. Strbac, "Voltage support from distribution level resources in South-East England," in 2018 IEEE Power and Energy Society General Meeting, August 2018.

[30] K. Clark, R. A. Walling, and N. W. Miller, "Solar photovoltaic (PV) plant models in pslf," in 2011 IEEE Power and Energy Society General Meeting, July 2011, pp. 1-5.

[31] J. M. MacDowell, K. Clark, N. W. Miller, and J. J. Sanchez-Gasca, "Validation of ge wind plant models for system planning simulations," in 2011 IEEE Power and Energy Society General Meeting, July 2011, pp. $1-8$.

[32] Y. Pipelzadeh, B. Chaudhuri, and T. C. Green, "Control coordination within a vsc hvdc link for power oscillation damping: A robust decentralized approach using homotopy," IEEE Transactions on Control Systems Technology, vol. 21, no. 4, pp. 1270-1279, July 2013

[33] D. Tzelepis, E. Tsotsopoulou, V. Nikolaidis, A. Dyko, V. Papaspiliotopoulos, Q. Hong, and C. Booth, "Impact of synchronous condensers on transmission line protection in scenarios with high penetration of renewable energy sources," in 2020 IET Developments in Power System Protection (DPSP), March 2020.

[34] P. Kansal and A. Bose, "Bandwidth and latency requirements for smart transmission grid applications," IEEE Transactions on Smart Grid, vol. 3, no. 3, pp. 1344-1352, Sep. 2012

[35] S. M. Blair, M. H. Syed, A. J. Roscoe, G. M. Burt, and J. Braun, "Measurement and analysis of pmu reporting latency for smart grid protection and control applications," IEEE Access, vol. 7, pp. 48689 $48698,2019$.

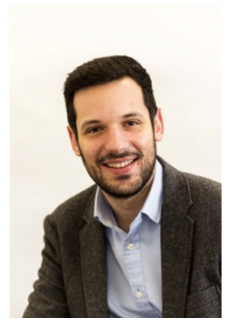

Anastasios Oulis Rousis (S'13-M'20) received the M.Eng. degree in Electrical and Computer Engineering from the National Technical University of Athens, Athens, Greece, in 2013, the M.Sc. degree in Wind Energy Systems from the University of Strathclyde, Glasgow, U.K., in 2014 and the Ph.D degree from Imperial College London in 2020. He is currently a Research Associate with Imperial College London. His expertise lies in power system optimization and renewable integration. His current work involves optimization of planning and operational models for transmission and distribution systems, including microgrids, while he investigates how flexible technologies can enhance the development of the future power systems.

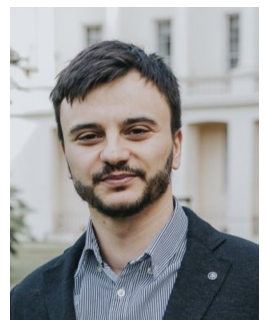

Dimitrios Tzelepis (S'13-M'17) received the B.Eng. (Hons) degree in electrical engineering from the Technological Education Institution of Athens, Athens, Greece, in 2013, and the M.Sc. degree in wind energy systems and the Ph.D. degree from the University of Strathclyde, Glasgow, U.K., in 2014 and 2017, respectively. $\mathrm{He}$ is currently a Postdoctoral Researcher with the Department of Electronic and Electrical Engineering, University of Strathclyde. His research interests lie within the area of power system protection, automation and control of future electricity grids, incorporating increased penetration of renewable energy sources and high voltage direct current interconnections. His main research methods include implementation of intelligent algorithms for protection, fault location and control applications including the utilisation of machine learning methods and advanced and intelligent signal processing techniques.

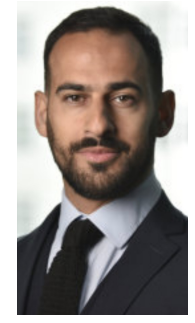

Yousef Pipelzadeh (S'09-M'12) is a Research Associate in Power Systems at Imperial College London and Head of Business Development for the Power System Technology Centre, a division of Manitoba Hydro International in the U.K. He is recognized by the British Standard Institute as a U.K. Principle Expert, and is an active member of the European Commission (Cenelec working group TC8X/WG 06) developing the guidelines and standards on HVDC Grids. He has particular interests in power system dynamics and offshore DC networks. $\mathrm{He}$ is an active member of IET and CIGRE.

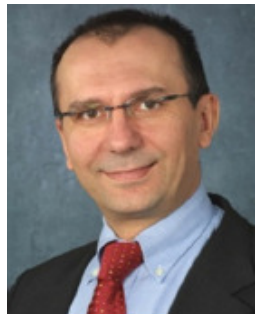

Goran Strbac (M'95) is a Professor of energy systems at Imperial College London, London, U.K. His current research is focused on modeling and optimization of economics and security of energy system operation and investment, energy infrastructure reliability, future energy markets, including integration of emerging technologies in supporting cost-effective evolution to smart low carbon energy future.

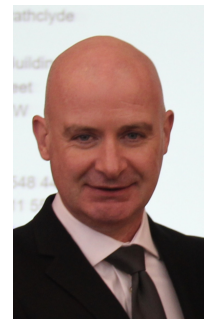

Campbell D. Booth received the B.Eng. and Ph.D. degrees in electrical and electronic engineering from the University of Strathclyde, Glasgow, Scotland, in 1991 and 1996, respectively. He is currently a Professor and the Head of Department for Electronic and Electrical Engineering, University of Strathclyde, Glasgow, U.K. His research interests include power system protection; plant condition monitoring and intelligent asset management; applications of intelligent system techniques to power system monitoring, protection, and control; knowledge management; and decision making.

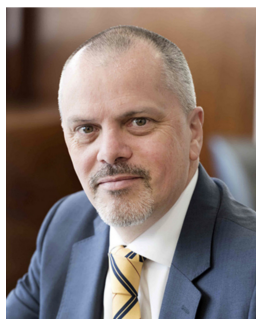

Timothy C. Green (M'89-SM'02-F'19) received a B.Sc. (Eng) (first class honours) from Imperial College London, UK in 1986 and a Ph.D. from HeriotWatt University, Edinburgh, UK in 1990 . He is a Professor of Electrical Power Engineering at Imperial College London, and Director of the Energy Futures Lab with a role fostering interdisciplinary energy research. His research interest is in using the flexibility of power electronics to accommodate new generation patterns and new forms of load, such as EV charging, as part of the emerging smart grid. In HVDC he has contributed converter designs that reduce losses while also providing control functions assist AC system integration. In distribution systems, he has pioneered the use of soft open points and the study of stability of grid connected inverters. Prof. Green is a Chartered Engineering the UK and a Fellow of the Royal Academy of Engineering. 